\title{
Hidden Charge Order in an Iron Oxide Square-Lattice Compound
}

\author{
Jung-Hwa Kim $\odot,{ }^{1, *}$ Darren C. Peets $\odot,{ }^{1,2,3,{ }^{*}}$ Manfred Reehuis $\odot,{ }^{4}$ Peter Adler $\odot,{ }^{5}$ Andrey Maljuk, ${ }^{1,6}$ \\ Tobias Ritschel, ${ }^{3}$ Morgan C. Allison, ${ }^{3}$ Jochen Geck, ${ }^{3,7}$ Jose R. L. Mardegan, ${ }^{8}$ Pablo J. Bereciartua Perez, \\ Sonia Francoual, ${ }^{8}$ Andrew C. Walters $\odot,{ }^{1,9}$ Thomas Keller, ${ }^{1,10}$ Paula M. Abdala, ${ }^{11}$ Philip Pattison, ${ }^{11,12}$ \\ Pinder Dosanjh, ${ }^{13}$ and Bernhard Keimer ${ }^{1, \dagger}$ \\ ${ }^{1}$ Max-Planck-Institut für Festkörperforschung, D-70569 Stuttgart, Germany \\ ${ }^{2}$ Ningbo Institute for Materials Technology and Engineering, Chinese Academy of Sciences, \\ Zhenhai, Ningbo, 315201 Zhejiang, China \\ ${ }^{3}$ Institut für Festkörper- und Materialphysik, Technische Universität Dresden, D-01069 Dresden, Germany \\ ${ }^{4}$ Helmholtz-Zentrum Berlin für Materialien und Energie, D-14109 Berlin, Germany \\ ${ }^{5}$ Max-Planck-Institut für Chemische Physik fester Stoffe, D-01187 Dresden, Germany \\ ${ }^{6}$ Leibniz Institut für Festkörper- und Werkstoffforschung, D-01171 Dresden, Germany \\ ${ }^{7}$ Würzburg-Dresden Cluster of Excellence ct.qmat, Technische Universität Dresden, 01062 Dresden, Germany \\ ${ }^{8}$ Deutsches Elektronen-Synchrotron DESY, Hamburg 22603, Germany \\ ${ }^{9}$ Diamond Light Source, Harwell Campus, Didcot OX11 ODE, United Kingdom \\ ${ }^{10}$ Max Planck Society Outstation at the Heinz Maier-Leibnitz Zentrum (MLZ), D-85748 Garching, Germany \\ ${ }^{11}$ SNBL at ESRF, BP 220, F-38042 Grenoble Cedex 9, France \\ ${ }^{12}$ Laboratory for Quantum Magnetism, École polytechnique fédérale de Lausanne (EPFL), \\ BSP-Dorigny, CH-1015 Lausanne, Switzerland \\ ${ }^{13}$ Department of Physics and Astronomy, University of British Columbia, Vancouver, BC, V6T 1Z1 Canada
}

(Received 7 April 2020; accepted 4 August 2021; published 27 August 2021)

\begin{abstract}
Since the discovery of charge disproportionation in the $\mathrm{FeO}_{2}$ square-lattice compound $\mathrm{Sr}_{3} \mathrm{Fe}_{2} \mathrm{O}_{7}$ by Mössbauer spectroscopy more than fifty years ago, the spatial ordering pattern of the disproportionated charges has remained "hidden" to conventional diffraction probes, despite numerous x-ray and neutron scattering studies. We have used neutron Larmor diffraction and Fe $K$-edge resonant x-ray scattering to demonstrate checkerboard charge order in the $\mathrm{FeO}_{2}$ planes that vanishes at a sharp second-order phase transition upon heating above $332 \mathrm{~K}$. Stacking disorder of the checkerboard pattern due to frustrated interlayer interactions broadens the corresponding superstructure reflections and greatly reduces their amplitude, thus explaining the difficulty of detecting them by conventional probes. We discuss the implications of these findings for research on "hidden order" in other materials.
\end{abstract}

DOI: 10.1103/PhysRevLett.127.097203

The term "hidden order" was coined for $d$ - and $f$-electron compounds that undergo a thermodynamic phase transition whose order parameter cannot be identified using conventional experimental methods [1-4]. The most prominent example is $\mathrm{URu}_{2} \mathrm{Si}_{2}$ whose hidden-order phase has confounded researchers for decades, despite numerous experimental and theoretical studies [1,2]. A lesser known, but equally puzzling case of hidden order has been found in the $\mathrm{FeO}_{2}$ square-lattice compound $\mathrm{Sr}_{3} \mathrm{Fe}_{2} \mathrm{O}_{7}$, whose electronically active $\mathrm{Fe}$ sites are accessible to Mössbauer spectroscopy. As early as 1966, [5,6] Mössbauer

Published by the American Physical Society under the terms of the Creative Commons Attribution 4.0 International license. Further distribution of this work must maintain attribution to the author(s) and the published article's title, journal citation, and DOI. Open access publication funded by the Max Planck Society. experiments on slightly oxygen deficient $\mathrm{Sr}_{3} \mathrm{Fe}_{2} \mathrm{O}_{7}$ revealed a disproportionation of the $\mathrm{Fe}^{4+}$ ions into nominal $\mathrm{Fe}^{3+}$ and $\mathrm{Fe}^{5+}$ valence states around room temperature. Numerous studies since then have confirmed a sharp phase transition at $T_{\mathrm{CO}}=340 \pm 10 \mathrm{~K}$ in stoichiometric $\mathrm{Sr}_{3} \mathrm{Fe}_{2} \mathrm{O}_{7}$, but no hints of a charge-ordering transition (such as a symmetry reduction or crystallographic site splitting) have ever been identified in diffraction data [6-12]. We have combined two advanced experimental methods, neutron Larmor diffraction (NLD) $[13,14]$ and resonant elastic x-ray scattering (REXS) $[15,16]$ at the Fe $K$ absorption edge, to resolve this long-standing conundrum. Specifically, we demonstrate checkerboard charge order in the $\mathrm{FeO}_{2}$ layers and show that the "invisibility" of charge ordering in $\mathrm{Sr}_{3} \mathrm{Fe}_{2} \mathrm{O}_{7}$ originates from frustration of the interactions between neighboring layers.

The impact of geometrical frustration on charge order has been widely investigated, beginning with the classical 
Verwey transition in magnetite $\left(\mathrm{Fe}_{3} \mathrm{O}_{4}\right)$ [17,18], and has recently been discussed for widely different solids ranging from metal oxides [19-21] to organic conductors [22,23], and in diverse contexts such as electronic ferroelectricity [19,20], superconductivity [21], quantum criticality [22], and phase-change memory applications [23].

$\mathrm{Sr}_{3} \mathrm{Fe}_{2} \mathrm{O}_{7}$ can serve as a model compound for frustrated charge order, because it is chemically stoichiometric and crystallizes in a body-centered tetragonal structure with $\mathrm{FeO}_{2}$ square-lattice bilayers [Fig. 1(a)]. This lattice architecture is common to many materials including $\mathrm{Sr}_{3} \mathrm{Ru}_{2} \mathrm{O}_{7}$, $\mathrm{La}_{2-2 x} \mathrm{Sr}_{1+2 x} \mathrm{Mn}_{2} \mathrm{O}_{7}$, and $\mathrm{La}_{2-x} \mathrm{Sr}_{x} \mathrm{CaCu}_{2} \mathrm{O}_{6+\delta}$, which have been intensely studied in relation to quantum criticality [24], magnetoresistance [25], and superconductivity [26]. Helical magnetic order due to competing exchange interactions between the $\mathrm{Fe}$ ions sets in at a much lower temperature $\left(T_{N}=115 \mathrm{~K}\right)$ and does not affect $T_{\mathrm{CO}}$ [27].
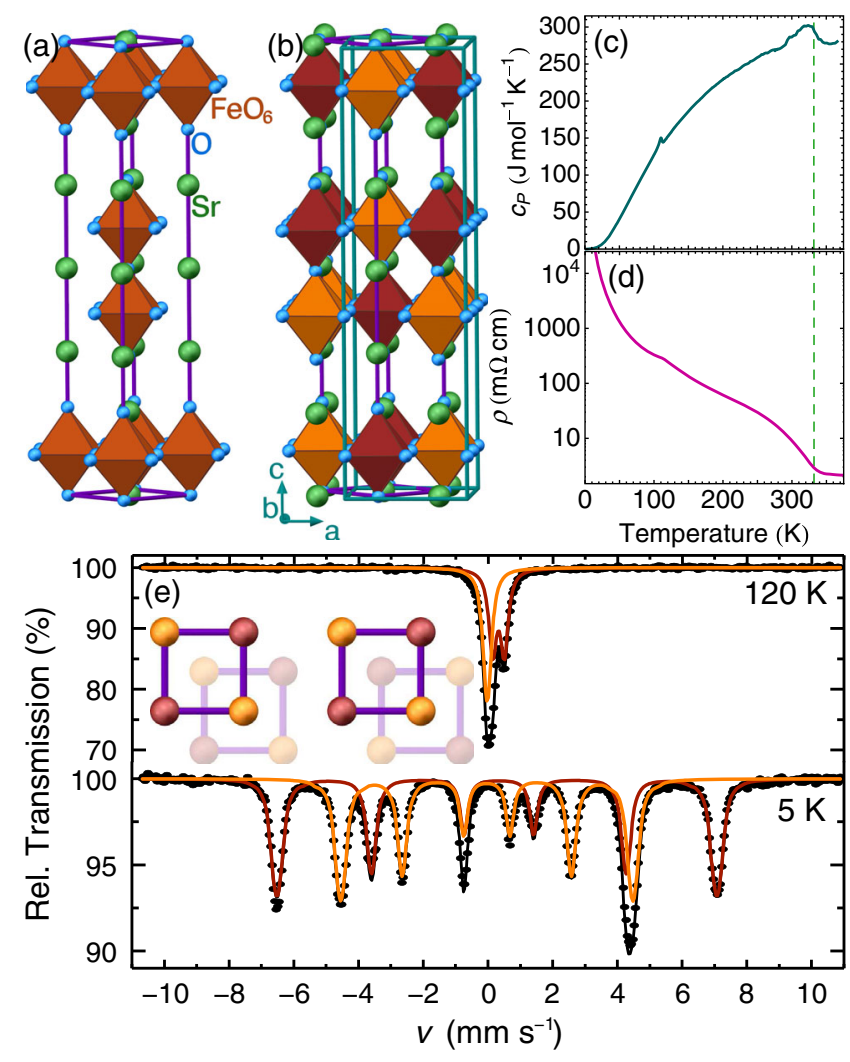

FIG. 1. Schematic crystal structures of (a) charge-disordered metallic and (b) charge-ordered insulating $\mathrm{Sr}_{3} \mathrm{Fe}_{2} \mathrm{O}_{7}$; colors indicate the $\mathrm{Fe}$ valence states. (c) Specific heat. An entropyconserving construction identifies a transition at $T_{\mathrm{CO}}=332 \mathrm{~K}$ (dashed line), consistent with transport and diffraction data. (d) In-plane resistivity showing a metal-insulator transition at $T_{\text {CO }}$. The anomalies at $T \sim 115 \mathrm{~K}$ in (c) and (d) are due to the onset of helical magnetic order. (e) Mössbauer spectra of $\mathrm{Sr}_{3} \mathrm{Fe}_{2} \mathrm{O}_{7}$ in the paramagnetic and magnetically ordered phases. The outer and inner components correspond to $\mathrm{Fe}^{3+}$ - and $\mathrm{Fe}^{5+}$ like sites, respectively [6-9,29]. Inset: two degenerate stacking patterns of $\mathrm{Fe}^{3+}$ - and $\mathrm{Fe}^{5+}$-like sites in adjacent bilayers.
Our crystallographic data imply that the cooperative Jahn-Teller effect is inactive and orbital order is absent in $\mathrm{Sr}_{3} \mathrm{Fe}_{2} \mathrm{O}_{7}$, in contrast to isoelectronic manganates such as $\mathrm{LaMnO}_{3}$, but similar to several rare-earth nickelates $R \mathrm{NiO}_{3}$ [28].

Frustration of the Coulomb interactions among valence electrons in $\mathrm{Sr}_{3} \mathrm{Fe}_{2} \mathrm{O}_{7}$ is caused by the body-centered stacking of $\mathrm{FeO}_{2}$ bilayers, with each $\mathrm{Fe}$ located directly above or below the center of a square iron-oxide plaquette in the adjacent bilayer [Figs. 1(b) and (e), inset]. We have found superstructure reflections indicative of checkerboard charge order in the $\mathrm{FeO}_{2}$ layers and demonstrated that stacking disorder due to frustrated interlayer coupling suppresses their amplitude below the detection limit of standard crystallographic probes. Interlayer frustration thus holds the key to the hidden-order conundrum in $\mathrm{Sr}_{3} \mathrm{Fe}_{2} \mathrm{O}_{7}$. Possible implications for other hidden-order materials are discussed.

High-quality single crystals of $\mathrm{Sr}_{3} \mathrm{Fe}_{2} \mathrm{O}_{7}$ were grown by the floating-zone technique $[12,36]$. In order to obtain full oxygen stoichiometry, single-crystalline rods were annealed under 5-6 kbar of oxygen pressure [29]. Specific heat data [Fig. 1(c)] demonstrate a second-order phase transition with a sizeable entropy release of $\sim 2.7 \mathrm{~J} / \mathrm{mol} \mathrm{K}$ at $T_{\mathrm{CO}}=332 \mathrm{~K}$. The transition is associated with a strong upturn in the in-plane resistivity [Fig. 1(d)], in agreement with previous reports $[11,12]$. To confirm charge ordering in our $\mathrm{Sr}_{3} \mathrm{Fe}_{2} \mathrm{O}_{7}$ samples, we conducted Mössbauer experiments on powdered crystals. The resulting spectra [Fig. 1(e)] reveal two components in the paramagnetic as well as in the magnetically ordered phases, indicating charge disproportionation of $\mathrm{Fe}^{4+}$ into $\mathrm{Fe}^{3+}$ - and $\mathrm{Fe}^{5+}$-like sites below $T_{\mathrm{CO}}$. (Note, however, that the high formal charge in $\mathrm{Fe}^{4+}$ compounds has to be understood in terms of negative charge transfer energy states, in which the excess holes and the electronic density modulation reside predominantly on the oxygen ligands [37,38].) The area ratio of $1: 1$ between the two subspectra confirms full oxidation of the sample and also indicates that the single iron site in the $I 4 / \mathrm{mmm}$ space group has split into two distinct sites with equal population, in agreement with prior work $[5-9,11]$.

Before addressing the charge-ordered phase, we used single-crystal neutron diffraction to verify the hightemperature crystal structure from which this order develops. Refinements [29] in the space group $I 4 / \mathrm{mmm}$ [Fig. 1(a)] showed no indications of any reduction in symmetry, in agreement with previous work $[6,10]$. The oxygen site bridging two adjacent $\mathrm{FeO}_{2}$ layers was refined to full occupancy as expected for stoichiometric $\mathrm{Sr}_{3} \mathrm{Fe}_{2} \mathrm{O}_{7}$.

We now turn to the crystal structure for $T<T_{\mathrm{CO}}$. We first note that neither our neutron diffraction data nor our high-resolution synchrotron x-ray powder pattern (Fig. 2) contained any additional primitive Bragg reflections to indicate a violation of the body-centering condition, nor 


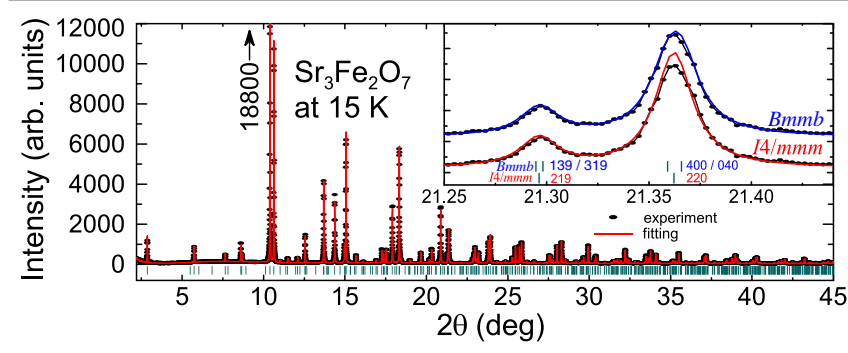

FIG. 2. High-resolution synchrotron $\mathrm{x}$-ray powder diffraction pattern of the charge-ordered phase at $T=15 \mathrm{~K}$. Inset: tetragonal (219) and (220) Bragg peaks, together with the results of refinements in the $I 4 / \mathrm{mmm}$ and $B m m b$ space groups.

any obvious splitting of peaks, in agreement with prior work that failed to detect any crystallographic signature of charge disproportionation $[6,10]$. There was a slight discrepancy between the synchrotron x-ray data and the $I 4 / \mathrm{mmm}$ refinements at a handful of peaks (inset of Fig. 2). To check whether anisotropic strain below $T_{\mathrm{CO}}$ could explain the peak profile broadening, we used a strain model (Laue class $4 / \mathrm{mmm}$ ) in the refinement, but the fit did not improve substantially. Nonetheless, these deviations alone were not compelling evidence for a change in crystal symmetry.

We therefore employed neutron Larmor diffraction on the TRISP spectrometer [39] at the Maier-LeibnitzZentrum in Garching, Germany. NLD is capable of detecting lattice parameters $d$ and their spread $\Delta d / d$ with a resolution better than $1 \times 10^{-4}$, independent of beam collimation and monochromaticity and of the crystal's mosaic spread $[13,14,29]$. Figure $3($ a) shows that $\Delta d / d$ of the tetragonal (220) Bragg reflection extracted from NLD increases sharply but continuously upon cooling below $T_{\mathrm{CO}}$, then saturates at a value of $4 \times 10^{-4}$, as expected for the order parameter of a structural phase transition. No comparable change of $\Delta d / d$ is observed along the $c$ axis (not shown), but the thermal expansion of the $c$-axis parameter extracted from the Larmor phase of the (0 0 10) reflection provides additional evidence of a continuous structural phase transition at $T_{\mathrm{CO}}$ [Fig. 3(b)].

The spread of the in-plane lattice parameters below $T_{\mathrm{CO}}$ revealed by NLD [Fig. 3(a)] and the slight discrepancy between the synchrotron x-ray data and $14 / \mathrm{mmm}$ refinements (Fig. 2) provide clues to the lattice structure in the charge-ordered state. Based on the observation of specific superstructure reflections indicative of a unit cell with doubled in-plane area (see below), we identify the orthorhombic space group $\mathrm{Bmmb}$ (an alternate setting of $\mathrm{Cmcm}$, No. 63) as the simplest crystallographic description compatible with our experimental data. Here, $\mathrm{Fe}^{3+}$ - and $\mathrm{Fe}^{5+}$ like sites in the $\mathrm{FeO}_{2}$ planes alternate in a checkerboard pattern within the plane and also within a bilayer unit along the $c$ axis [Fig. 1(b)]. The checkerboard pattern is analogous to the charge-ordering patterns in the pseudocubic
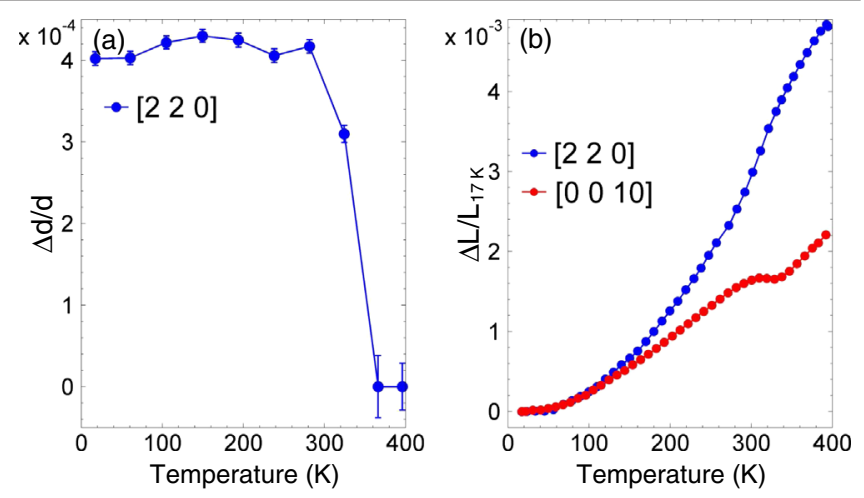

FIG. 3. Neutron Larmor diffraction results. (a) Splitting of the in-plane lattice parameters, $\Delta d / d=(b-a) / a$, extracted from the tetragonal (220) Bragg peak as a function of temperature $T$. In the analysis, the intrinsic peak width of $\Delta Q / Q=4.8 \times 10^{-4}$ at $400 \mathrm{~K}$ was kept constant at all $T$. (b) Thermal expansion extracted from the cumulative Larmor phase of the tetragonal (220) and (0010) Bragg peaks, relative to the phase at $T=17 \mathrm{~K}$.

perovskite $\mathrm{CaFeO}_{3}$, where $\mathrm{Fe}^{3+}$ - and $\mathrm{Fe}^{5+}$-like sites alternate in all three directions [40].

Rietveld refinements in $B m m b$ produced lattice constants $a=5.43050(3) \AA, \quad b=5.43287(3) \AA$, and $c=20.12134(6) \AA$. We note that the difference between $a$ and $b,(b-a) / a=4.0 \times 10^{-4}$, is in quantitative agreement with the independent neutron Larmor diffraction results for the in-plane $\Delta d / d$ [Fig. 3(a)]. Complete tables of the resulting structural parameters are given in the Supplemental Material [29]. The refinement does not indicate any rotations of the $\mathrm{FeO}_{6}$ octahedra such as those observed in nearly isostructural $\mathrm{Sr}_{3} \mathrm{Ru}_{2} \mathrm{O}_{7}[41,42]$ and in $\mathrm{CaFeO}_{3}$ [40]. Since substantial rotations and distortions of the $\mathrm{FeO}_{6}$ octahedra are present in $\mathrm{CaFeO}_{3}$ even above $T_{\mathrm{CO}}$, the splitting of its orthorhombic Bragg reflections $\Delta Q / Q$ is $\sim 20$ times that of the tetragonal peaks in $\mathrm{Sr}_{3} \mathrm{Fe}_{2} \mathrm{O}_{7}$ for $T<T_{\mathrm{CO}}$. Charge order then manifests as a spectral-weight shift between the split peaks, which is readily resolved by standard diffraction probes [40].

To search for weak superstructure reflections that are allowed in $\mathrm{Bmmb}$ but not in $I 4 / \mathrm{mmm}$, we first investigated a $\sim 10 \mu$ m-diameter $\mathrm{Sr}_{3} \mathrm{Fe}_{2} \mathrm{O}_{7}$ single crystal at the BM01A beamline at the European Synchrotron Radiation Facility, using the wavelength $0.6973 \AA$. In $B m m b$, such reflections occur at $(h, 0, l)$ for $h+k=2 n$ and $(0, k, l)$ for $l=2 n$. In $I 4 / \mathrm{mmm}$, these have noninteger indices $(h / 2, k / 2, l)$ and are therefore forbidden. We found no intensity at the position of any superstructure reflection, and were able to place an upper bound of $10^{-5}$ on the ratio of the peak intensities $I(h k l) /[I(115)+I(-115)]$ (in the orthorhombic setting), which should be of order $10^{-4}$ according to our refinement [29]. If our space group assignment is correct, this finding implies that the superstructure reflections are broadened by disorder so that their amplitude is reduced below the detection limit. 

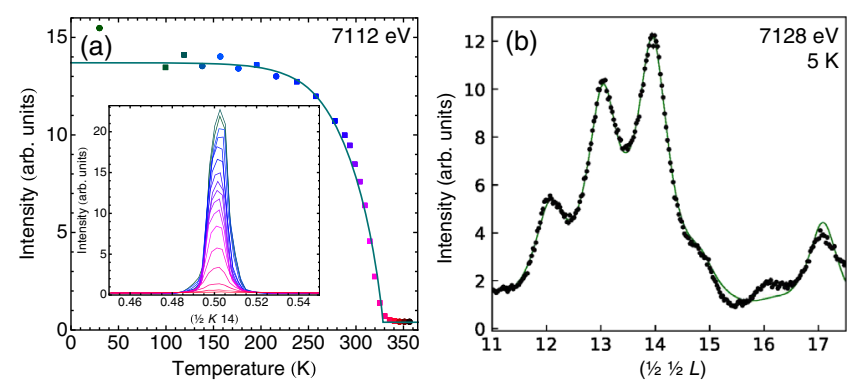

FIG. 4. (a) Temperature dependence of the integrated intensity of the $\left(\frac{1}{2} \frac{1}{2} 14\right)$ superstructure reflection measured by Fe $K$-edge REXS slightly off resonance (photon energy $7112 \mathrm{eV}$ ). The line is a guide to the eye. Inset: in-plane $K$ scans at temperatures marked by squares in the main panel, demonstrating the absence of $T$-dependent shifts or broadening of this reflection. (b) $L$ scan on resonance at $T=30 \mathrm{~K}$. The line is the result of a calculation that considers only the Fe sites, assuming differing charge. The $\mathrm{Fe}$ positions were taken from the crystallographic refinement [29], and only the width, overall intensity, and the imbalance between the population of orthorhombic twin domains (45:55\%) were fitted. Reciprocal-space locations refer to the high-temperature $I 4 / \mathrm{mmm}$ cell. $\left(\frac{1}{2} \frac{1}{2} 14\right)$ in $I 4 / \mathrm{mmm}$ is equivalent to (1 0 14)/(0 1 14) in Bmmb.

To enhance the sensitivity to the diffraction signal from a charge modulation on the Fe sites, we performed singlecrystal REXS $[15,16]$ measurements at the Fe $K$ edge (photon energy $7128 \mathrm{eV}$ ) at beamline P09 at the PETRA-II synchrotron, Deutsches Elektronen-Synchrotron, in Hamburg, Germany [43]. The incoming polarization was perpendicular to the scattering plane, and the outgoing polarization was not analyzed. As shown in Fig. 4, REXS indeed enables the detection of superstructure peaks at the positions predicted for the space group Bmmb. The $\left(\frac{1}{2}, \frac{1}{2}, l\right)$ reflections (in $I 4 / \mathrm{mmm}$ notation) are direct manifestations of the unit cell doubling due to checkerboard charge order. The intensity of the superstructure reflections decreases continuously with increasing $T$ and vanishes at $T=T_{\mathrm{CO}}$ [Fig. 4(a)]. Figure 4 represents our most crucial result, as it demonstrates that the checkerboard ordering pattern is correct and has temperature dependence consistent with Mössbauer (Fig. 1) and neutron Larmor diffraction (Fig. 3) data.

In addition to the data shown in Fig. 4, we surveyed $~ 50$ reciprocal-space positions, including primitive reflections forbidden in Bmmb [29]. In particular, superstructure peaks having a temperature dependence consistent with those shown in Fig. 4 were also found at $\left(\frac{1}{2}, \frac{3}{2}, l\right)$ positions (tetragonal cell) for both even and odd $l$, but not at tetragonal $(0,0,2 n+1)$ or $(1,1,2 n+1)$, as expected for $B m m b$. The structure factor of these reflections is roughly consistent with a model that only considers the contribution of the iron atoms resulting from the structural refinement [Fig. 4(b)]. In this model, the structure factor of the $\left(\frac{1}{2}, \frac{1}{2}, l\right)$ reflections shown in Fig. 4(b) is given by
$4 f_{1} \sin \left(2 \pi z_{1} l\right)+4 f_{2} \sin \left(2 \pi z_{2} l\right)$, where $f_{1,2}$ are the form factors of inequivalent $\mathrm{Fe}$ ions, and $z_{1} \approx-z_{2} \approx 0.097$ are their $c$-axis positions measured from the center of the unit cell [Fig. 1(b), center] [29]. Some deviations from the model calculations are apparent, possibly indicating a contribution from $\mathrm{FeO}_{6}$ octahedral distortions which modulate the $\mathrm{Fe} 4 p$ intermediate state of $K$-edge REXS, as recently found in experiments on nickel oxides [44]. Since the positional parameters of the $\mathrm{O}$ atoms cannot be accurately extracted from the structural refinement [29], comprehensive modeling of the REXS intensity on and off resonance goes beyond the scope of the current Letter and will be the subject of future work.

Here we emphasize that the observation of the superstructure reflections and the corresponding extinction rules complete the space-group assignment in the charge-ordered state. The low-temperature space group has to accommodate iron ions in two different valence states as found by the Mössbauer experiments. Since the phase transition at $T_{\mathrm{CO}}$ is second order, we considered subgroups of $I 4 / \mathrm{mmm}$ with two crystallographically different Fe sites. The observation of peaks at half-integer positions in $h$ and $k$ requires a unit cell with a doubled in-plane area. This requirement leads to Fmmm and its direct subgroups. Out of these, Cmme and Cmce do not support charge order. Fmmm itself and its subgroups Fmm2, F222, Ccce, and Cccm can also be ruled out, because $\left(\frac{1}{2}, 0, l\right)$ and $\left(0, \frac{1}{2}, l\right)$ reflections were found for both odd and even $l$ [Fig. 4(b)]. Likewise, the nonobservation of $(1,1,7)$ and $(1,1,9)$ (again in $I 4 / \mathrm{mmm}$ notation) excludes $C 2 / \mathrm{m}$. This leaves $\mathrm{Bmmm}$ and $\mathrm{Bmmb}$, which feature in-plane checkerboards with uniform and alternating stacking within a unit cell (and corresponding structure factors with cosinelike and sinelike dependence on $l$ ), respectively. The experimentally observed sinelike structure factor [Fig. 4(b)] singles out Bmmb, which is also favored by electrostatic and structural considerations. Deviations from orthorhombic symmetry (such as a monoclinic distortion) were not found outside the experimental uncertainty.

The REXS data yield insight into the origin of the "invisibility" of the charge-ordered state to standard diffraction probes. Whereas the width of the superstructure reflections is resolution-limited in the $\mathrm{FeO}_{2}$ planes (which implies a lower bound of 100 tetragonal lattice spacings on the in-plane correlation length), the reflections are broadened into diffuse streaks along the $c$ axis. From their momentum width, we infer a domain size of $\sim 0.65$ lattice spacings along $c$. Because of the corresponding reduction of the peak amplitude by about 2 orders of magnitude, the superstructure peaks are below the detection threshold of standard neutron and nonresonant x-ray scattering. The correlation along the $c$ axis is strong within, but weak between bilayer units, so that the order can be regarded as quasi-two-dimensional. Nonetheless, the observation of well-defined superstructure peaks implies that the space 
group is correct and the stacking is not random. It is interesting to point out that the Ising symmetry of the charge-order parameter allows a finite-temperature phase transition in two dimensions [45], which helps explain the sharp transition in the thermodynamic, transport, and diffraction data even in the presence of substantial stacking disorder.

The insights gained from our resolution of the longstanding $\mathrm{Sr}_{3} \mathrm{Fe}_{2} \mathrm{O}_{7}$ conundrum provide interesting perspectives for research on hidden order in other compounds. First, we note that Mössbauer spectroscopy provides a sensitive, direct probe of the charge and spin density modulation in $\mathrm{Sr}_{3} \mathrm{Fe}_{2} \mathrm{O}_{7}$. Without Mössbauer data (which are only obtainable on a small number of compounds with Mössbauer-active elements), the origin of the prominent phase transition in $\mathrm{Sr}_{3} \mathrm{Fe}_{2} \mathrm{O}_{7}$ would have been far less evident. This is the case, for instance, for the layered iridates with reported hidden-order transitions [4] as well as the archetypical hidden-order compound $\mathrm{URu}_{2} \mathrm{Si}_{2}$ [1,2], which also crystallize in $14 / \mathrm{mmm}$ with the same bodycentered stacking pattern of electronically active atoms (i.e., iridium or uranium) as in $\mathrm{Sr}_{3} \mathrm{Fe}_{2} \mathrm{O}_{7}$.

We also note that the orthorhombic distortion breaks the fourfold rotational symmetry of the tetragonal host lattice, and is thus expected to induce twofold angular modulations in thermodynamic and transport quantities if domain averaging can be avoided (by reducing the sample volume or by applying external strain). Such modulations have indeed been identified in experiments by Okazaki and coworkers on $\mathrm{URu}_{2} \mathrm{Si}_{2}$ [46], and were attributed to "nematic" order, that is, a state with broken rotational symmetry that maintains the translational symmetry of the host lattice. Our observation of the orthorhombic supercell in $\mathrm{Sr}_{3} \mathrm{Fe}_{2} \mathrm{O}_{7}$ suggests an alternative explanation of this behavior. The possible sensitivity of the orthorhombic domain size along the $c$ axis to cooling protocols or defects might help explain why the experiment of Okazaki et al. has been difficult to reproduce [2].

In summary, our NLD and REXS experiments have resolved the 50-year-old puzzle of "hidden" charge order in $\mathrm{Sr}_{3} \mathrm{Fe}_{2} \mathrm{O}_{7}$. The results highlight the need for further investigations of the influence of frustration and disorder on experimental observables in hidden-order phases of other materials. Finally, we point out that the high ordering temperature of $\mathrm{Sr}_{3} \mathrm{Fe}_{2} \mathrm{O}_{7}$ might enable device applications akin to those recently proposed for organic compounds with frustrated charge order [23].

We thank an anonymous referee for insightful comments on an earlier version of this manuscript. We acknowledge funding from the Deutsche Forschungsgemeinschaft (DFG, German Research Foundation), Collaborative Research Center TRR 80 (Project No. 107745057), and through projects $\mathrm{C} 03$ and $\mathrm{C} 06$ of the Collaborative Research Center SFB 1143 (Project No. 247310070); the National Natural Science Foundation of China (Grant No. 11674367), and the Zhejiang Provincial Natural Science Foundation (Grant No. LZ18A040002). D. C.P. is supported by the Chinese Academy of Sciences through 2018PM0036. The authors are grateful to the groups of R. Dinnebier, R. Kremer, and L. Schröder, and the staff of beamline P02.1 at PETRA-III for experimental support. We thank HZB for the allocation of neutron diffraction beamtime; DESY (Hamburg, Germany), a member of the Helmholtz Association HGF, for resonant diffraction beamtime; the European Synchrotron Radiation Facility for the provision of synchrotron facilities and access to beamlines BM01A and BM01B; and the Heinz Maier-Leibnitz Zentrum (MLZ), Garching, Germany, for use of the TRISP spectrometer at FRM II.

*These authors contributed equally to this work. †b.keimer@fkf.mpg.de

[1] J. A. Mydosh and P. M. Oppeneer, Colloquium: Hidden order, superconductivity, and magnetism: The unsolved case of $\mathrm{URu}_{2} \mathrm{Si}_{2}$, Rev. Mod. Phys. 83, 1301 (2011).

[2] J. A. Mydosh, P. M. Oppeneer, and P. S. Riseborough, Hidden order and beyond: An experimental-Theoretical overview of the multifaceted behavior of $\mathrm{URu}_{2} \mathrm{Si}_{2}$, J. Phys. Condens. Matter 32, 143002 (2020).

[3] A. S. Cameron, G. Friemel, and D. S. Inosov, Multipolar phases and magnetically hidden order: Review of the heavyfermion compound $\mathrm{Ce}_{1-x} \mathrm{La}_{x} \mathrm{~B}_{6}$, Rep. Prog. Phys. 79, 066502 (2016).

[4] G. Cao and P. Schlottmann, The challenge of spin-orbittuned ground states in iridates: A key issues review, Rep. Prog. Phys. 81, 042502 (2018).

[5] P. K. Gallagher, J. B. MacChesney, and D. N. E. Buchanan, Mössbauer effect in the system $\mathrm{Sr}_{3} \mathrm{Fe}_{2} \mathrm{O}_{6-7}$, J. Chem. Phys. 45, 2466 (1966).

[6] S. E. Dann, M. T. Weller, D. B. Currie, M. F. Thomas, and A.D. Al-Rawwas, Structure and magnetic properties of $\mathrm{Sr}_{2} \mathrm{FeO}_{4}$ and $\mathrm{Sr}_{3} \mathrm{Fe}_{2} \mathrm{O}_{7}$ studied by powder neutron diffraction and Mössbauer spectroscopy, J. Mater. Chem. 3, 1231 (1993).

[7] P. Adler, Electronic state, magnetism, and electrical transport behavior of $\mathrm{Sr}_{3-x} A_{x} \mathrm{Fe}_{2} \mathrm{O}_{7}(x \leq 0.4, A=\mathrm{Ba}, \mathrm{La}), \mathrm{J}$. Solid State Chem. 130, 129 (1997).

[8] H. Kobayashi, M. Kira, H. Onodera, T. Suzuki, and T. Kamimura, Electronic state of $\mathrm{Sr}_{3} \mathrm{Fe}_{2} \mathrm{O}_{7-y}$ studied by specific heat and Mössbauer spectroscopy, Physica (Amsterdam) 237B-238B, 105 (1997), Proceedings of the Yamada Conference XLV, the International Conference on the Physics of Transition Metals.

[9] P. Adler, U. Schwarz, K. Syassen, G. K. Rozenberg, G. Y. Machavariani, A.P. Milner, M.P. Pasternak, and M. Hanfland, Collapse of the charge disproportionation and covalency-driven insulator-metal transition in $\mathrm{Sr}_{3} \mathrm{Fe}_{2} \mathrm{O}_{7}$ under pressure, Phys. Rev. B 60, 4609 (1999).

[10] K. Mori, T. Kamiyama, H. Kobayashi, S. Torii, F. Izumi, and H. Asano, Crystal structure of $\mathrm{Sr}_{3} \mathrm{Fe}_{2} \mathrm{O}_{7-\delta}$, J. Phys. Chem. Solids 60, 1443 (1999). 
[11] K. Kuzushita, S. Morimoto, S. Nasu, and S. Nakamura, Charge disproportionation and antiferromagnetic order of $\mathrm{Sr}_{3} \mathrm{Fe}_{2} \mathrm{O}_{7}$, J. Phys. Soc. Jpn. 69, 2767 (2000).

[12] D. C. Peets, J.-H. Kim, P. Dosanjh, M. Reehuis, A. Maljuk, N. Aliouane, C. Ulrich, and B. Keimer, Magnetic phase diagram of $\mathrm{Sr}_{3} \mathrm{Fe}_{2} \mathrm{O}_{7-\delta}$, Phys. Rev. B 87, 214410 (2013).

[13] M. T. Rekveldt, T. Keller, and R. Golub, Larmor precession, a technique for high-sensitivity neutron diffraction, Europhys. Lett. 54, 342 (2001).

[14] T. Keller, M. T. Rekveldt, and K. Habicht, Neutron Larmor diffraction measurement of the lattice-spacing spread of pyrolytic graphite, Appl. Phys. A 74, S127 (2002).

[15] J. E. Lorenzo, Y. Joly, D. Mannix, and S. Grenier, Charge order as seen by resonant (elastic) X-ray scattering, Europhys. J. Special Topics 208, 121 (2012).

[16] J. Fink, E. Schierle, E. Weschke, and J. Geck, Resonant elastic soft x-ray scattering, Rep. Prog. Phys. 76, 056502 (2013).

[17] F. Walz, The Verwey transition-A topical review, J. Phys. Condens. Matter 14, R285 (2002).

[18] J. P. Attfield, Charge ordering in transition metal oxides, Solid State Sci. 8, 861 (2006).

[19] S. Ishihara, Electronic ferroelectricity and frustration, J. Phys. Soc. Jpn. 79, 011010 (2010).

[20] N. Ikeda, T. Nagata, J. Kano, and S. Mori, Present status of the experimental aspect of $R \mathrm{Fe}_{2} \mathrm{O}_{4}$ study, J. Phys. Condens. Matter 27, 053201 (2015).

[21] K. Jiang, S. Zhou, and Z. Wang, Textured electronic states of the triangular-lattice Hubbard model and $\mathrm{Na}_{x} \mathrm{CoO}_{2}$, Phys. Rev. B 90, 165135 (2014).

[22] L. Cano-Cortés, A. Ralko, C. Février, J. Merino, and S. Fratini, Geometrical frustration effects on charge-driven quantum phase transitions, Phys. Rev. B 84, 155115 (2011).

[23] H. Oike, F. Kagawa, N. Ogawa, A. Ueda, H. Mori, M. Kawasaki, and Y. Tokura, Phase-change memory function of correlated electrons in organic conductors, Phys. Rev. B 91, 041101(R) (2015).

[24] R. A. Borzi, S. A. Grigera, J. Farrell, R. S. Perry, S. J. S. Lister, S. L. Lee, D. A. Tennant, Y. Maeno, and A. P. Mackenzie, Formation of a nematic fluid at high fields in $\mathrm{Sr}_{3} \mathrm{Ru}_{2} \mathrm{O}_{7}$, Science 315, 214 (2007).

[25] T. Kimura, Y. Tomioka, H. Kuwahara, A. Asamitsu, M. Tamura, and Y. Tokura, Interplane tunneling magnetoresistance in a layered manganite crystal, Science 274, 1698 (1996).

[26] R. J. Cava, B. Batlogg, R. B. van Dover, J. J. Krajewski, J. V. Waszczak, R. M. Fleming, W. F. Peck Jr, L. W. Rupp Jr, P. Marsh, A. C. W. P. James, and L. F. Schneemeyer, Superconductivity at $60 \mathrm{~K}$ in $\mathrm{La}_{2-x} \mathrm{Sr}_{x} \mathrm{CaCu}_{2} \mathrm{O}_{6}$ : The simplest double-layer cuprate, Nature (London) 345, 602 (1990).

[27] J.-H. Kim, A. Jain, M. Reehuis, G. Khaliullin, D. C. Peets, C. Ulrich, J. T. Park, E. Faulhaber, A. Hoser, H. C. Walker, D. T. Adroja, A. C. Walters, D. S. Inosov, A. Maljuk, and B. Keimer, Competing Exchange Interactions on the Verge of a Metal-Insulator Transition in the Two-Dimensional Spiral Magnet $\mathrm{Sr}_{3} \mathrm{Fe}_{2} \mathrm{O}_{7}$, Phys. Rev. Lett. 113, 147206 (2014).

[28] I. I. Mazin, D. I. Khomskii, R. Lengsdorf, J. A. Alonso, W. G. Marshall, R. M. Ibberson, A. Podlesnyak, M. J. Martínez-Lope, and M. M. Abd-Elmeguid, Charge Ordering as Alternative to Jahn-Teller Distortion, Phys. Rev. Lett. 98, 176406 (2007).

[29] See Supplemental Material at http://link.aps.org/ supplemental/10.1103/PhysRevLett.127.097203, including Refs. [30-35], for additional experimental details, crystallographic parameters, and Mössbauer results.

[30] The XTAL3.4 User's Manual, edited by S. R. Hall, G. S. D. King, and J. M. Stewart (Lamb Print, University of Western Australia, Perth, 1995).

[31] International Tables of Crystallography, Vol. C, edited by E. Prince (Kluwer Academic Publishers, Dordrecht, 2006).

[32] J. Rodríguez-Carvajal, Recent advances in magnetic structure determination by neutron powder diffraction, Physica (Amstetrdam) 192B, 55 (1993).

[33] Z. Klencsár, MossWinn-methodological advances in the field of Mössbauer data analysis, Hyperfine Interact. 217, 117 (2013).

[34] M. Takano, N. Nakanishi, Y. Takeda, S. Naka, and T. Takada, Charge disproportionation in $\mathrm{CaFeO}_{3}$ studied with the Mössbauer effect, Mater. Res. Bull. 12, 923 (1977).

[35] G. Demazeau, B. Buffat, F. Ménil, L. Fournès, M. Pouchard, J. M. Dance, P. Fabritchnyi, and P. Hagenmuller, Characterization of six-coordinated iron (V) in an oxide lattice, Mater. Res. Bull. 16, 1465 (1981).

[36] A. Maljuk, J. Strempfer, C. Ulrich, M. Sofin, L. Capogna, C. Lin, and B. Keimer, Growth of $\mathrm{Sr}_{3} \mathrm{Fe}_{2} \mathrm{O}_{7-x}$ single crystals by the floating zone method, J. Cryst. Growth 273, 207 (2004).

[37] A. E. Bocquet, A. Fujimori, T. Mizokawa, T. Saitoh, H. Namatame, S. Suga, N. Kimizuka, Y. Takeda, and M. Takano, Electronic structure of $\mathrm{SrFe}^{4+} \mathrm{O}_{3}$ and related $\mathrm{Fe}$ perovskite oxides, Phys. Rev. B 45, 1561 (1992).

[38] R. J. Green, M. W. Haverkort, and G. A. Sawatzky, Bond disproportionation and dynamical charge fluctuations in the perovskite rare-earth nickelates, Phys. Rev. B 94, 195127 (2016).

[39] T. Keller and B. Keimer, TRISP: Three axes spin echo spectrometer, J. Large-Scale Res. Facil. 1, A37 (2015).

[40] P. M. Woodward, D. E. Cox, E. Moshopoulou, A. W. Sleight, and S. Morimoto, Structural studies of charge disproportionation and magnetic order in $\mathrm{CaFeO}_{3}$, Phys. Rev. B 62, 844 (2000).

[41] H. Shaked, J. D. Jorgensen, O. Chmaissem, S. Ikeda, and Y. Maeno, Neutron diffraction study of the structural distortions in $\mathrm{Sr}_{3} \mathrm{Ru}_{2} \mathrm{O}_{7}$, J. Solid State Chem. 154, 361 (2000).

[42] R. Kiyanagi, K. Tsuda, N. Aso, H. Kimura, Y. Noda, Y. Yoshida, S.-I. Ikeda, and Y. Uwatoko, Investigation of the structure of single crystal $\mathrm{Sr}_{3} \mathrm{Ru}_{2} \mathrm{O}_{7}$ by neutron and convergent beam electron diffractions, J. Phys. Soc. Jpn. 73, 639 (2004).

[43] J. Strempfer, S. Francoual, D. Reuther, D. K. Shukla, A. Skaugen, H. Schulte-Schrepping, T. Krachta, and H. Franz, Resonant scattering and diffraction beamline P09 at PETRA III, J. Synchrotron Radiat. 20, 541 (2013).

[44] Y. Lu, A. Frano, M. Bluschke, M. Hepting, S. Macke, J. Strempfer, P. Wochner, G. Cristiani, G. Logvenov, H.-U. Habermeier, M. W. Haverkort, B. Keimer, and E. Benckiser, Quantitative determination of bond order and lattice dis- 
tortions in nickel oxide heterostructures by resonant $\mathrm{x}$-ray scattering, Phys. Rev. B 93, 165121 (2016).

[45] S. Onoda, Y. Motome, and N. Nagaosa, Two-Dimensional Charge Order in Layered 2-1-4 Perovskite Oxides, Phys. Rev. Lett. 92, 236403 (2004).
[46] R. Okazaki, T. Shibauchi, H. J. Shi, Y. Haga, T. D. Matsuda, E. Yamamoto, Y. Onuki, H. Ikeda, and Y. Matsuda, Rotational symmetry breaking in the hidden-order phase of $\mathrm{URu}_{2} \mathrm{Si}_{2}$, Science 331, 439 (2011). 